CREADORES DE EMPRESAS DE BASE TECNOLÓGICA (SPIN-OFF). IDENTIFICANDO SU PERFIL ÓPTIMO EN MÉXICO.

\title{
CREADORES DE EMPRESAS DE BASE TECNOLÓGICA (SPIN-OFF). IDENTIFICANDO SU PERFIL ÓPTIMO EN MÉXICO
}

\section{CREATORS OF TECHNOLOGY-BASED COMPANIES (SPIN-OFF). IDENTIFYING YOUR OPTIMAL PROFILE IN MEXICO}

José Vili Martínez González*, Vili Aldebarán Martínez García**

*Doctor en Administración. Ajedrez Consultores. Secretario General del COLPARMEX. Ecatepec, Estado de México. ORCID: http://orcid.org/0000-0002-0978-5778.

**Joven Investigador de Ajedrez Consultores. Ecatepec, Estado de México. ORCID: http://orcid.org/00000002-3901-0121.

Dirección para recibir correspondencia: ajedrezconsultores@hotmail.com

Fecha de recibido: 21 de agosto de 2019

Fecha de aceptación: 19 de septiembre de 2019

DOI: https://doi.org/10.19136/hitos.a25n73.3532

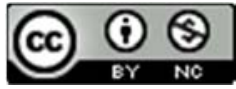

MARTÍNEZ-GONZÁLEZ J. V., MARTÍNEZ-GARCÍA V. A.

SEPTIEMBRE-DICIEMBRE 2019. Año 25, Número 73, Págs. 315-333 
CREADORES DE EMPRESAS DE BASE TECNOLÓGICA (SPIN-OFF). IDENTIFICANDO SU PERFIL ÓPTIMO EN MÉXICO.

\title{
RESUMEN
}

Existe un creciente interés en los países de economías desarrolladas por fomentar las denominadas Empresas de Base Tecnológica (EBT) también llamadas Spin-Off y, si bien, en México desde hace varios años se han emprendido acciones para buscar fomentar este tipo de empresas, los resultados no han sido los esperados, ciertamente, esto se debe a causas multifactoriales, siendo uno de ellos la falta de un perfil óptimo para este tipo de empresarios.

OBJETIVO: Identificar un perfil óptimo para quienes crean EBT en México.

MATERIAL Y MÉTODO: Se realizó una investigación de tipo descriptiva, transeccional, utilizando el instrumento PSO1 para identificar los principales factores considerados de gran importancia para consolidar EBT, utilizando una muestra de sujeto tipo integrada por 26 participantes.

RESULTADOS: Fue factible identificar un perfil óptimo para quienes crean EBT en México.

CONCLUSIONES: El tener un perfil óptimo brinda información que contribuye para que quienes van a crear o quienes ya tienen una EBT en operaciones identifiquen sus áreas de oportunidad coadyuvando a sus posibilidades de tener éxito, al identificar en forma puntual conocimientos y habilidades que han sido señaladas como importantes, por quienes formaron parte de la muestra.

PALABRAS CLAVE: Empresas de Base Tecnológica. Perfil óptimo. Spin-Off.

\begin{abstract}
There is a growing interest in the countries of developed economies to promote the so-called Technological Base Companies (EBT) also called Spin-Off and, although, in Mexico for several years actions have been taken to seek to promote these types of companies, Results have not been as expected, certainly, this is due to multifactorial causes, one of them being the lack of an optimal profile for this type of entrepreneurs.
\end{abstract}

OBJECTIVE: Identify an optimal profile for those who create EBT in Mexico. 
CREADORES DE EMPRESAS DE BASE TECNOLÓGICA (SPIN-OFF). IDENTIFICANDO SU PERFIL ÓPTIMO EN MÉXICO.

MATERIAL AND METHOD: A descriptive, transectional investigation was carried out, using PSO1 instrument to identify the main factors considered of great importance to consolidate EBT, using a sample of type subject with a total of 26 participants.

RESULTS: It was feasible to identify an optimal profile for those who create EBT in Mexico.

CONCLUSIONS: Having an optimal profile provides information that contributes so that those who are going to create or who already have an EBT in operations identify their areas of opportunity contributing to their chances of success, by identifying in a timely manner knowledge and skills that have been indicated as important, for those who were part of the sample.

KEY WORDS: Technology Based Companies. Optimal profile. Spin-Off.

\section{INTRODUCCIÓN}

El nuevo entorno empresarial exige la creación de empresas de alto valor que no solo participen en la generación de empleos, sino que, además, al considerar la naturaleza de estas empresas, genere dentro de su plantilla puestos de trabajo para personal con un alto perfil profesional considerando que, anexo a ello, al ofrecer soluciones tecnológicas satisfagan nichos de mercados que en algunos casos no existían en forma previa coadyuvando a mejorar la productividad y creando una derrama económica. En este artículo se trata en particular de las empresas conocidas como Empresas de Base Tecnológica (EBT o Spin-Off).

Considerando información de la Organización para la Cooperación y el Desarrollo Económico (OCDE), es factible mencionar que en los últimos años una gran cantidad de países que integran dicha organización y las economías emergentes más importantes han fomentado políticas públicas que permitan su creación y desarrollo, así como diferentes mecanismos que contribuyan a las mismas; sin embargo, la situación en México resulta no ser tan alentadora.

Si bien, el gobierno mexicano ha creado políticas y mecanismos para fomentar el desarrollo de las EBT, la OCDE menciona que (salvo algunos casos), todas las medidas de apoyo se han destinado a proyectos de innovación, pertenecen a empresas que ya están en funcionamiento, 
CREADORES DE EMPRESAS DE BASE TECNOLÓGICA (SPIN-OFF). IDENTIFICANDO SU PERFIL ÓPTIMO EN MÉXICO.

dejando con ello de lado la creación de nuevas empresas basadas en la tecnología; lo anterior, deriva en una falla esencial en la búsqueda de crear un entorno adecuado para las EBT.

Aunado a lo anterior, es importante el señalar que la OCDE ha identificado tres factores críticos que se suman a las dificultades existentes para la apertura de este tipo de empresas:

1.- Mercados financieros poco desarrollados;

2.- Pobre desarrollo y poca valoración de los activos intangibles;

3.- Obstáculos para el desarrollo de nuevas empresas basadas en ciencia y tecnología a partir de las instituciones públicas de investigación.

Anexo a los anteriores factores, existe uno que resulta tan discreto que no se piensa en él hasta que ya se enfrentan sus consecuencias, la falta de un perfil óptimo que permita orientar a quienes van a participar en la creación de EBT sobre las habilidades y conocimientos que deberán dominar además de los técnicos y propios de la tecnología involucrada y, es aquí donde surge la aportación del trabajo al buscar identificar precisamente ese perfil.

Es importante el conceptualizar el concepto de EBT o Spin-Off considerando que:

Para el Centro Europeo de Empresas e Innovación, (2012, p.9), el término Spin-Off es un término anglosajón cuyo significado engloba aquellas empresas creadas en el seno de otra empresa ya existente, pública o privada, que actúa como matriz y que sirve de apoyo en los primeros pasos de su trayectoria. Estas empresas suelen nacer en muchos casos, de las universidades o centros de investigación públicos, si bien con el tiempo adquieren independencia jurídica, técnica y comercial constituyéndose como empresas de nueva creación.

Para García Colina, et. al., (2017, p. 46) "son iniciativas empresariales promovidas por miembros de la comunidad universitaria. Se caracterizan por basar su actividad en la explotación de nuevos procesos, productos o servicios a partir del conocimiento adquirido y los resultados obtenidos en la propia universidad".

Finalmente, el Consejo Nacional de Ciencia y Tecnología (CONACyT) citado en Sanchéz Esgua (2016, p. 7), identifica a las "Empresas de Base Tecnológica (EBT) como unidades de negocios productoras de bienes y servicios cuya competitividad depende del diseño, desarrollo y MARTíNEZ-GONZÁLEZ J. V., MARTÍNEZ-GARCÍA V. A. 
CREADORES DE EMPRESAS DE BASE TECNOLÓGICA (SPIN-OFF). IDENTIFICANDO SU PERFIL ÓPTIMO EN MÉXICO.

producción de nuevos productos o procesos innovadores, a través de la aplicación sistemática e intensiva de conocimientos científicos y tecnológicos".

Por ello, resulta factible identificar que estas empresas nacen de desarrollos tecnológicos innovadores, teniendo esto en consideración, es importante visualizar cuál es la realidad de la innovación en México y un indicador, por lo que es importante conocer el nivel de patentes solicitadas y obtenidas.

Considerando las cifras del Instituto Mexicano de la Propiedad Intelectual (IMPI) tenemos que aun estando en México la cantidad de patentes solicitadas (considerando la nacionalidad de los solicitantes) representa solo el $7.52 \%$ del total en el 2016 , el $7.76 \%$ en el 2017 y se incrementa a un $9.47 \%$ en el 2018, (ver tabla 1 ).

Tabla 1

Solicitudes de patente por nacionalidad

\begin{tabular}{|c|c|c|c|c|c|c|c|c|c|c|c|}
\hline Año & Total & México & Alemania & $\begin{array}{l}\text { Estados } \\
\text { Unidos }\end{array}$ & Francia & Italia & Japón & $\begin{array}{l}\text { Reino } \\
\text { Unido }\end{array}$ & España & Suiza & $\begin{array}{l}\text { Otros } \\
\text { países }\end{array}$ \\
\hline 2016 & 17,413 & 1,310 & 1,153 & 8,262 & 594 & 301 & 1,181 & 319 & 204 & 968 & 3,121 \\
\hline 2017 & 17,184 & 1,334 & 1,106 & 8,370 & 585 & 287 & 1,274 & 379 & 186 & 897 & 2,766 \\
\hline 2018 & 16,424 & 1,555 & 1,155 & 7,173 & 520 & 307 & 1,191 & 423 & 197 & 905 & 2,998 \\
\hline
\end{tabular}

Fuente: Elaboración propia, empleando datos de Instituto Mexicano de la Propiedad Industrial, (2018).

En lo relativo a la cantidad de patentes otorgadas considerando la nacionalidad de quien las solicitó la situación tampoco es alentadora ya que en el 2016 representó solo el 4.92\%, para el 2017 la cifra disminuyó al 4.78\% recuperándose en el 2018 al llegar a un 5.12\%, (ver tabla 2).

Tabla 2

Patentes otorgadas por nacionalidad del titular

\begin{tabular}{|c|c|c|c|c|c|c|c|c|c|}
\hline Año & Total & México & Alemania & $\begin{array}{c}\text { Estados } \\
\text { Unidos }\end{array}$ & Francia & Japón & $\begin{array}{c}\text { Reino } \\
\text { Unido }\end{array}$ & Suiza & $\begin{array}{c}\text { Otros } \\
\text { países }\end{array}$ \\
\hline 2016 & 8,657 & 426 & 653 & 4,032 & 380 & 566 & 196 & 497 & 1,907 \\
\hline 2017 & 8,510 & 407 & 625 & 3,950 & 359 & 570 & 179 & 492 & 1,928 \\
\hline 2018 & 8,921 & 457 & 676 & 4,176 & 328 & 615 & 142 & 427 & 2,100 \\
\hline
\end{tabular}

Fuente: Elaboración propia, empleando datos de Instituto Mexicano de la Propiedad Industrial, (2018). 
CREADORES DE EMPRESAS DE BASE TECNOLÓGICA (SPIN-OFF). IDENTIFICANDO SU PERFIL ÓPTIMO EN MÉXICO.

Otro indicador que resulta revelador es la gran disparidad que existe entre las diferentes entidades federativas en lo que refiere a las solicitudes de invenciones por parte de mexicanos, considerando los años de 2016, 2017 y 2018 se encontró que la entidad con mayor número de solicitudes es la Ciudad de México con el $22.55 \%, 22.22 \%$ y $21.74 \%$ respectivamente, seguido a la distancia por Jalisco con 13.55\%, 16.03\%, y 14.99\%, (ver tabla 3).

Tabla 3

Solicitudes de invenciones de mexicanos por entidad federativa

\begin{tabular}{|c|c|c|c|c|c|c|c|c|c|c|c|c|}
\hline \multirow[t]{2}{*}{$\begin{array}{c}\text { Entidad } \\
\text { Federativa } \\
\end{array}$} & \multicolumn{4}{|c|}{ Enero-diciembre 2016} & \multicolumn{4}{|c|}{ Enero-diciembre de 2017} & \multicolumn{4}{|c|}{ Enero-diciembre de 2018} \\
\hline & Patentes & $\begin{array}{c}\text { Diseños } \\
\text { industriales }\end{array}$ & $\begin{array}{l}\text { Modelos de } \\
\text { utilidad }\end{array}$ & Total & Patentes & $\begin{array}{c}\text { Diseños } \\
\text { industriales }\end{array}$ & $\begin{array}{c}\text { Modelos de } \\
\text { utilidad }\end{array}$ & Total & Patentes & $\begin{array}{c}\text { Diseños } \\
\text { industriales }\end{array}$ & $\begin{array}{l}\text { Modelos de } \\
\text { utilidad }\end{array}$ & Total \\
\hline Aguascalientes & 21 & 75 & 0 & 96 & 8 & 35 & 5 & 48 & 10 & 27 & 2 & 39 \\
\hline Baja California & 20 & 26 & 14 & 60 & 25 & 24 & 22 & 71 & 28 & 14 & 14 & 56 \\
\hline $\begin{array}{l}\text { Baja California } \\
\text { Sur }\end{array}$ & 2 & 0 & 2 & 4 & 6 & 1 & 1 & 8 & 5 & 1 & 1 & 7 \\
\hline Campeche & 10 & 2 & 4 & 16 & 3 & 0 & 12 & 15 & 8 & 1 & 13 & 22 \\
\hline $\begin{array}{l}\text { Ciudad de } \\
\text { México }\end{array}$ & 308 & 387 & 109 & 804 & 333 & 342 & 104 & 779 & 345 & 381 & 115 & 841 \\
\hline Chiapas & 23 & 5 & 7 & 35 & 4 & 2 & 5 & 11 & 20 & 3 & 23 & 46 \\
\hline Chihuahua & 42 & 15 & 16 & 73 & 32 & 15 & 15 & 62 & 66 & 42 & 22 & 130 \\
\hline Coahuila & 49 & 26 & 20 & 95 & 51 & 36 & 25 & 112 & 77 & 27 & 47 & 151 \\
\hline Colima & 11 & 1 & 9 & 21 & 5 & 0 & 0 & 5 & 4 & 8 & 3 & 15 \\
\hline Durango & 9 & 3 & 12 & 24 & 6 & 2 & 2 & 10 & 18 & 8 & 7 & 33 \\
\hline $\begin{array}{l}\text { Estado de } \\
\text { México }\end{array}$ & 94 & 150 & 61 & 305 & 80 & 148 & 56 & 284 & 85 & 124 & 72 & 281 \\
\hline Guanajuato & 71 & 263 & 32 & 366 & 78 & 305 & 28 & 411 & 67 & 287 & 26 & 380 \\
\hline Guerrero & 2 & 2 & 0 & 4 & 1 & 0 & 4 & 5 & 3 & 2 & 2 & 7 \\
\hline Hidalgo & 14 & 5 & 6 & 25 & 29 & 14 & 6 & 49 & 31 & 9 & 7 & 47 \\
\hline Jalisco & 152 & 236 & 95 & 483 & 194 & 294 & 74 & 562 & 219 & 276 & 85 & 580 \\
\hline Michoacán & 12 & 9 & 8 & 29 & 11 & 21 & 9 & 41 & 16 & 12 & 4 & 32 \\
\hline Morelos & 36 & 14 & 9 & 59 & 32 & 13 & 7 & 52 & 40 & 26 & 6 & 72 \\
\hline Nayarit & 1 & 7 & 1 & 9 & 3 & 1 & 3 & 7 & 4 & 1 & 5 & 10 \\
\hline Nuevo León & 87 & 173 & 79 & 339 & 78 & 210 & 50 & 338 & 100 & 141 & 81 & 322 \\
\hline Oaxaca & 15 & 1 & 2 & 18 & 15 & 1 & 0 & 16 & 18 & 1 & 1 & 20 \\
\hline Puebla & 84 & 62 & 12 & 158 & 90 & 38 & 14 & 144 & 119 & 43 & 25 & 187 \\
\hline Querétaro & 57 & 45 & 23 & 125 & 62 & 50 & 15 & 127 & 34 & 42 & 25 & 101 \\
\hline Quintana Roo & 5 & 6 & 9 & 20 & 11 & 4 & 7 & 22 & 12 & 4 & 12 & 28 \\
\hline San Luis Potosí & 21 & 32 & 4 & 57 & 9 & 3 & 1 & 13 & 13 & 29 & 1 & 43 \\
\hline Sinaloa & 27 & 21 & 5 & 53 & 26 & 5 & 10 & 41 & 51 & 26 & 3 & 80 \\
\hline Sonora & 28 & 7 & 11 & 46 & 41 & 16 & 7 & 64 & 35 & 8 & 9 & 52 \\
\hline Tabasco & 16 & 4 & 2 & 22 & 16 & 1 & 4 & 21 & 8 & 3 & 1 & 12 \\
\hline Tamaulipas & 23 & 18 & 21 & 62 & 26 & 10 & 21 & 57 & 45 & 23 & 45 & 113 \\
\hline Tlaxcala & 3 & 3 & 2 & 8 & 3 & 3 & 5 & 11 & 4 & 11 & 3 & 18 \\
\hline Veracruz & 34 & 16 & 14 & 64 & 20 & 11 & 11 & 42 & 49 & 6 & 17 & 72 \\
\hline Yucatán & 27 & 28 & 20 & 75 & 24 & 22 & 14 & 61 & 18 & 29 & 19 & 66 \\
\hline Zacatecas & 3 & 8 & 3 & 14 & 5 & 8 & 4 & 17 & 3 & 14 & 12 & 29 \\
\hline
\end{tabular}

Fuente: Elaboración propia, empleando datos de Instituto Mexicano de la Propiedad Industrial, (2018).

Finalmente, considerando que uno de los factores que permitiría crear una Spin-Off es el contar con la patente resulta importante el visualizar quiénes son los principales titulares de las mismas MARTÍNEZ-GONZÁLEZ J. V., MARTÍNEZ-GARCÍA V. A.

SEPTIEMBRE-DICIEMBRE 2019. Año 25, Número 73, Págs. 315-333 
CREADORES DE EMPRESAS DE BASE TECNOLÓGICA (SPIN-OFF). IDENTIFICANDO SU PERFIL ÓPTIMO EN MÉXICO.

considerando que su país de origen sea México y es factible observar que en el 2018 la UNAM encabeza la lista seguido por el Centro de Investigación y de Estudios Avanzados del I.P.N., (ver tabla 4).

Tabla 4

Principales titulares de patentes en México, siendo su país de origen México de enero a diciembre del 2018

\begin{tabular}{lc}
\hline Universidad Nacional Autónoma de México & 44 \\
Centro de Investigación y de Estudios Avanzados del I.P.N.* & 38 \\
Instituto Politécnico Nacional & 23 \\
Universidad Autónoma de Nuevo León & 23 \\
Instituto Tecnológico y de Estudios Superiores de Monterrey & 15 \\
Benemérita Universidad Autónoma de Puebla & 15 \\
Centro de Investigación y Asistencia en Tecnología y Diseño del Estado de Jalisco, A.C. & 13 \\
Instituto Mexicano del Petróleo & 8 \\
Universidad Autónoma Metropolitana & 7 \\
Centro de Investigación en Química Aplicada & 5 \\
Instituto Nacional de Astrofísica, Óptica y Electrónica & 5 \\
Mabe, S.A. de C.V. & 5 \\
Universidad de Guanajuato & 5
\end{tabular}

44

Fuente: Elaboración propia, empleando datos de Instituto Mexicano de la Propiedad Industrial, (2018).

Si bien, las cifras anteriores brindan un claro panorama de cómo se desarrolla el tema de las patentes e invenciones en México; por ello, es importante el contrastar estas cifras con el comportamiento que se tiene a nivel mundial, considerando cifras de World Intellectual Property Organization, (2018), menciona que en el 2017 se presentaron en todo el mundo 3,17 millones de solicitudes de patente, recordando que en México en ese año se presentaron solo 17,184 solicitudes. Anexo a lo anterior, en ese mismo año la oficina de Propiedad Intelectual de China recibió 1,380,000 solicitudes de patentes, seguidos por la oficina de Estados Unidos de América con 606.956 y Japón con 318.479.

Si a lo anterior, se anexa que el Índice Global de Innovación (Dutta, Lanvin, \& Wunsch-Vincent, 2019) coloca a México en el lugar 56 (ocupando la tercera posición en América Latina), es de MARTÍNEZ-GONZÁLEZ J. V., MARTÍNEZ-GARCÍA V. A. 
CREADORES DE EMPRESAS DE BASE TECNOLÓGICA (SPIN-OFF). IDENTIFICANDO SU PERFIL ÓPTIMO EN MÉXICO.

comprender que el panorama resulta altamente retador. Es precisamente en este entorno que la presente investigación busca coadyuvar a quienes van a emprender o ya cuentan con una EBT en marcha.

\section{MATERIAL Y MÉTODO}

Se realizó una investigación de tipo descriptiva, transeccional, utilizando instrumento PSO1 para identificar los principales factores considerados de gran importancia para consolidar EBT, utilizando una muestra de sujeto tipo con un total de 26 participantes.

En la presentación del instrumento se especificó:

a) El objetivo del estudio;

b) Su carácter que sería anónimo;

c) Que la información sería tratada en forma confidencial;

d) Que la información sería analizada en conjunto para publicaciones científicas y de difusión en congresos especializados;

e) Que en ningún caso se publicarían datos individuales.

f) Que no se recogerían datos personales como nombre o algún dato que permitirá su identificación.

No existe conflicto de interés en la realización de este estudio.

Se utilizó el PSO1 para identificar los factores que los participantes del estudio determinaron como las principales áreas de oportunidad, en lo relativo al perfil óptimo que debería tener quien emprende o dirige una Spin-Off, este instrumento cuenta con un Alpha de 0.7522 y está constituido por 25 reactivos en tres dimensiones, las cuales son:

- Conocimientos;

- Habilidades;

- Actitudes. 
CREADORES DE EMPRESAS DE BASE TECNOLÓGICA (SPIN-OFF). IDENTIFICANDO SU PERFIL ÓPTIMO EN MÉXICO.

Es importante resaltar que, este instrumento fue diseñado para identificar las habilidades y conocimientos a empresarios que ya se encuentran con una EBT en operación o que están en la etapa de iniciar operaciones.

Los criterios de inclusión considerados fueron:

a) Personas laboralmente activas;

b) Edad de 20 a 70 años;

c) Que estén emprendiendo o ya tengan una EBT en funcionamiento.

Los criterios de exclusión considerados fueron:

a) Personas menores de 19 años o mayores de 71;

b) Que no hubieran completado correctamente el test;

c) Que no estén emprendiendo o no tengan una EBT en funcionamiento.

Se trató de una muestra de sujeto tipo con un total de 26 cuestionarios.

Conceptualización de las variables consideradas:

Conocimiento: Se considera como el resultado de la comprensión de la información por medio del aprendizaje. Siendo el conjunto de hechos, principios, teorías y prácticas que relacionados con un campo de trabajo o estudio en particular.

Habilidad: Se considera a la capacidad de aplicar conocimientos y técnicas para hacer en forma correcta una actividad con facilidad.

Actitudes: Se considera como la disposición para presentar una conducta ante una condición específica.

Perfil óptimo: Se considera como el conjunto de conocimientos, habilidades y actitudes que han sido identificados como factores que coadyuvan al éxito de los creadores de empresas de base tecnológica. 
CREADORES DE EMPRESAS DE BASE TECNOLÓGICA (SPIN-OFF). IDENTIFICANDO SU PERFIL ÓPTIMO EN MÉXICO.

\section{RESULTADOS}

La mayor parte de los conocimientos y habilidades identificados como áreas de oportunidad por quienes se encuentran transitando de la investigación básica a la investigación aplicada (inicio de las EBT), también fueron mencionadas por quienes ya se encuentran con empresas en pleno desarrollo experimental; es decir, quienes ya tienen empresas en funciones. Para visualizar mejor estas etapas se consideran los conceptos mencionados en la Organización para la Cooperación y el Desarrollo Económico, (2018).

Investigación básica, investigación aplicada y desarrollo experimental. La investigación básica consiste en trabajos experimentales o teóricos que se emprenden fundamentalmente para obtener nuevos conocimientos acerca de los fundamentos de fenómenos y hechos observables, sin intención de otorgarles ninguna aplicación o utilización determinada. La investigación aplicada consiste también en trabajos originales realizados para adquirir nuevos conocimientos, pero está dirigida fundamentalmente hacia un objetivo práctico específico. El desarrollo experimental consiste en trabajos sistemáticos fundamentados en los conocimientos existentes obtenidos a partir de la investigación o la experiencia práctica, que se dirigen a producir nuevos productos o procesos, o a mejorar los productos o procesos que ya existen (p. 47).

En este punto es factible mencionar que quienes se encuentran inmersos en el campo de las ciencias, frecuentemente están acostumbrados a obtener recursos y tratar con equipos de trabajo para la primera etapa, sin embargo, las habilidades, conocimientos y actitudes para tener éxito en las dos siguientes con mira a innovar un producto o servicio resultan distintas encontrando que el perfil óptimo de un creador de EBT (Spin-Off) sugiere (sin limitar) las siguientes:

Perfil óptimo sugerido para los creadores de Empresas de Base Tecnológica (Spin-Off).

- Recursos humanos;

- Comunicación efectiva;

- Administración del tiempo;

- Negociación;

- Desarrollo de planes de negocio;

- Mercadotecnia; 
CREADORES DE EMPRESAS DE BASE TECNOLÓGICA (SPIN-OFF). IDENTIFICANDO SU PERFIL ÓPTIMO EN MÉXICO.

- Aspectos legales en general;

- Requisitos legales para poner en marcha un negocio;

- Requisitos legales del producto o servicio.

Ciertamente resulta interesante el notar que en el perfil obtenido las habilidades, conocimientos y actitudes se entrelazan en forma tal que la frontera entre ellas se desdibuja, de tal forma que, lo que algunos teóricos mencionarían como el tener conocimiento del área de recursos humanos en el caso de quienes emprenden una Empresa de Base Tecnológica también adquiere características de habilidad pues, en esta área, deben tener la posibilidad de entrenar a otros en conjunto con una actitud correcta para incentivar (motivar) a su equipo de trabajo. Por lo anterior, se presentan los elementos que integran el perfil óptimo con el deseo de que sirvan de guía sin buscar ser exhaustivos o limitantes.

En la figura 1, es factible observar los nueve elementos que conforman el perfil óptimo identificado para los creadores de Empresas de Base Tecnológica. Con base en la información obtenida en la investigación, es posible mencionar que el desarrollo de estos nueve tópicos coadyuvará en un mejor desempeño para quienes emprenden una EBT, recordando que, en gran medida, quienes se aventuran en este tipo de emprendimiento están más próximos a las áreas académicas, de investigación y, su entorno de confort se encuentra en laboratorios y universidades por lo que, enfrentarse a un mundo empresarial puede resultar en el mejor de los casos, simplemente apabullante. El considerar los siguientes tópicos en su formación resultará no solo enriquecedor en forma general, sino que, permitirá una mejor toma de decisiones al tener elementos para evaluar diferentes caminos de acción. Por ejemplo, el conocer el marco legal para el registro de una marca sin convertirse en un experto de la materia (ya que esto implicaría una inversión importante de tiempo), permitirá que tome decisiones más adecuadas y, que al momento de elegir el despacho o gestores para dicho trámite sepa que puede pedir, así como los tiempos de espera y el alcance de protección de la misma, permitiendo, además, el tener un diálogo más eficiente con quien realice dicho registro.

Lo anterior, adquiere una dimensión de suma importancia ya que, muchas veces el entusiasmo de llevar a cabo una idea que tiene el potencial para resolver un problema crítico para la sociedad se estrella con la "intransigencia" de un verificador que por no tener un plano de ruta de evacuación está dispuesto a clausurar el edificio donde está iniciando la EBT. Es importante comprender que existen normas y reglamentos en el país, que tienen un motivo de existencia y MARTíNEZ-GONZÁLEZ J. V., MARTÍNEZ-GARCíA V. A. 
CREADORES DE EMPRESAS DE BASE TECNOLÓGICA (SPIN-OFF). IDENTIFICANDO SU PERFIL ÓPTIMO EN MÉXICO.

son de carácter obligatorio por lo que, se deben cumplir en forma previa al arranque de la empresa. En este sentido, no se trata de que los creadores de EBT tengan conocimientos totales sobre las gestiones de dichos permisos, sino que sean capaces de identificar qué permisos se deben cumplir y obtener la asesoría y apoyo suficiente para cumplir con los mismos. En este punto, surge la importancia de las incubadoras de empresas considerando que son lugares donde se les puede facilitar a los creadores de EBT lugares físicos para iniciar sus trámites, así como diferentes tipos de servicios necesarios para poner en marcha su negocio.

A lo largo de las siguientes figuras, se hará mención en forma más puntual a cada uno de los conceptos, exceptuando comunicación efectiva, administración del tiempo y desarrollo de planes de negocio ya que estas se identificaron con un concepto unitario de alto impacto perfectamente diferenciado de otros, verbigracia, el concepto de comunicación se refiere a la adquisición y desarrollo de conocimientos, habilidades y actitudes para lograr un proceso efectivo de comunicación el cual fue señalado por el 69.23\%, en tanto que la administración del tiempo fue señalado por el $92.31 \%$ y el desarrollo de plan de negocios por el $100 \%$.

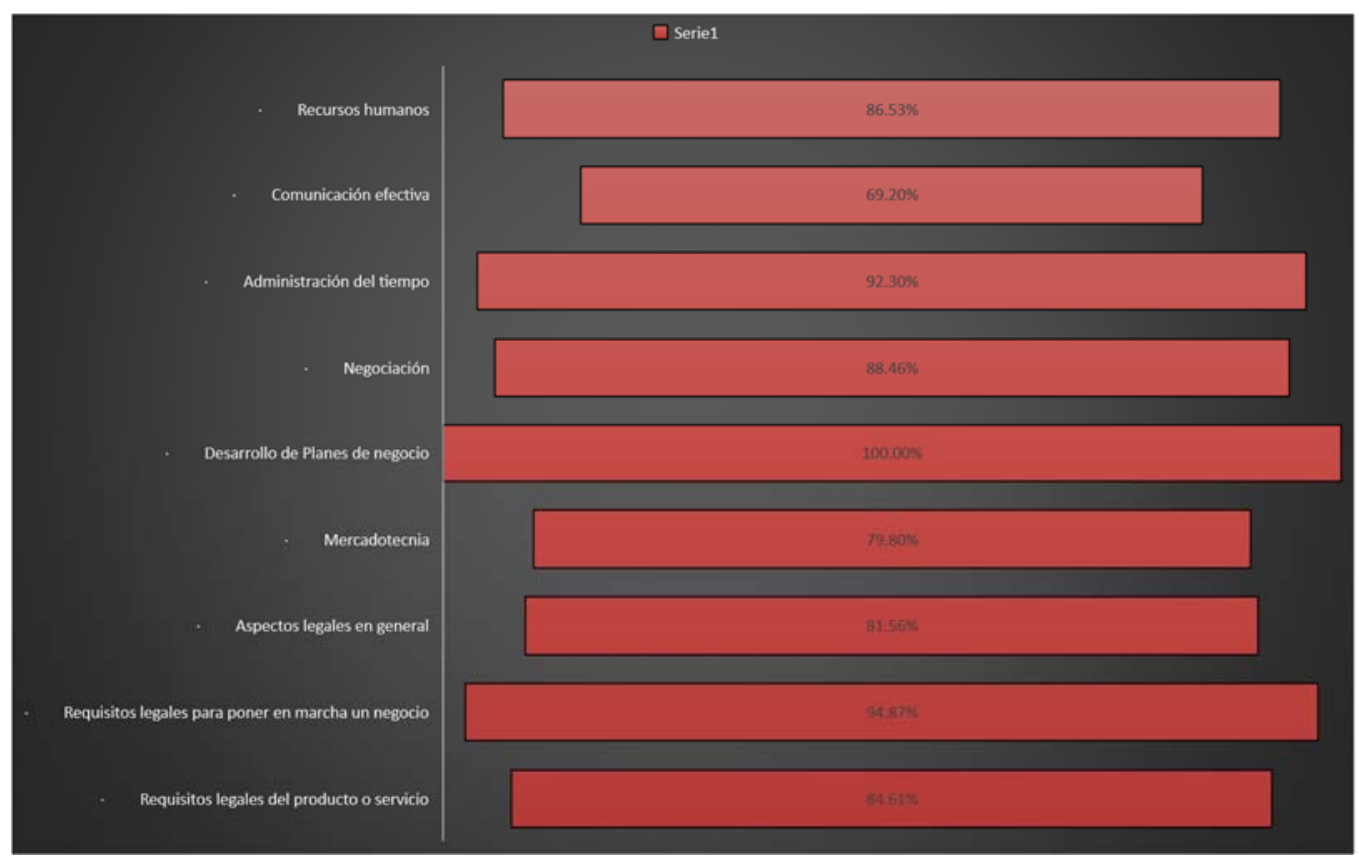

Figura 1. Conocimientos, habilidad y aptitudes identificadas como parte del perfil óptimo para creadores de Spin-Off.

Fuente: Elaboración propia.

En la figura 2, se incluyen los conceptos identificados como importantes para los creadores de EBT en lo relativo al tema de los recursos humanos, cabe hacer mención que, al pasar de dirigir MARTÍNEZ-GONZÁLEZ J. V., MARTÍNEZ-GARCÍA V. A. 
CREADORES DE EMPRESAS DE BASE TECNOLÓGICA (SPIN-OFF). IDENTIFICANDO SU PERFIL ÓPTIMO EN MÉXICO.

equipos cuyo pago y soporte recaían en las instituciones donde realizaban sus investigaciones y, que ahora, al tomar personalidad jurídica propia, deben ser responsables de sueldos, salarios, prestaciones, contrataciones, despidos y todas las actividades de las organizaciones en marcha.

Ciertamente son múltiples las actividades que deben considerar, sin la intensión de convertirse en expertos en la materia pues lo más viable es contratar a un equipo que se encargue de ello, encontrando que, en forma recurrente, durante las etapas primarias sean los propios creadores de la EBT quienes se encarguen de solventar estos temas, de tal forma que, una vez que se haya logrado la consolidación y crecimiento de la organización estos asuntos se puedan delegar a expertos en la materia siendo deseable que se sigan involucrando tanto en la supervisión de los resultados de la gestión de los temas relacionados con los recursos humanos, como en los procesos que conlleven relación con personal crítico (investigadores de primer nivel para la organización y aquellos que tengan acceso y conozcan el "know how" de los procesos claves).

Como puede observarse, en el tema de los recursos humanos los integrantes de la muestra seleccionada referenciaron como un tópico de importancia crítica lo relativo a los aspectos legales con un $100 \%$, las técnicas para reclutar y seleccionar con un $80.77 \%$, las técnicas de incentivación/motivación con un $92.31 \%$ y la capacitación y entrenamiento con el 73.08\%.

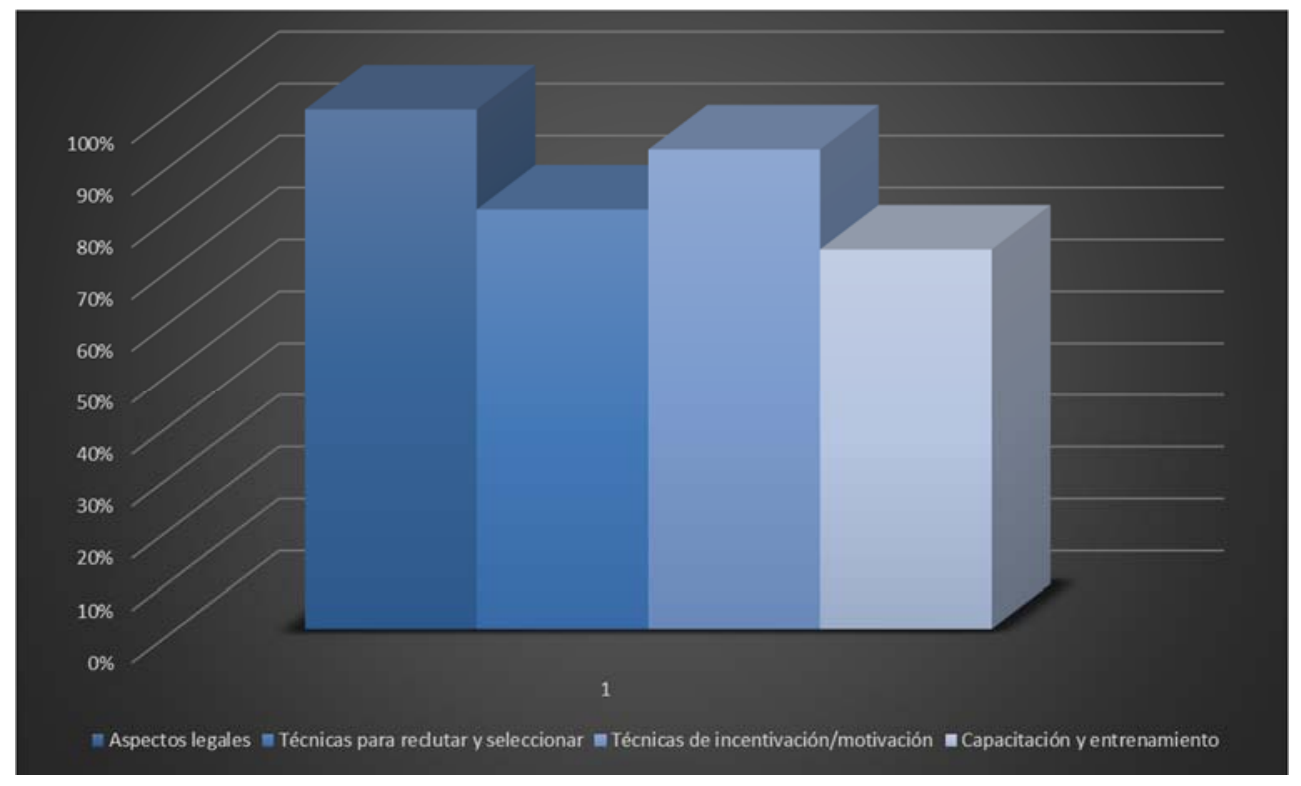

Figura 2. Recursos humanos.

Fuente: Elaboración propia. 
CREADORES DE EMPRESAS DE BASE TECNOLÓGICA (SPIN-OFF). IDENTIFICANDO SU PERFIL ÓPTIMO EN MÉXICO.

Una de las habilidades más críticas referenciadas se refirió al tema de negociación. Esta simple habilidad resultó crítica para el éxito de quienes participaron en la investigación (en este punto surgió la duda de poder localizar a quienes no hubieran logrado concretar su EBT y obtener su opinión al respecto; es decir, preguntarles si consideran que la falta negociación fue un concomitante que impidió que se lograra consolidar su empresa).

En la figura 3, los integrantes de la muestra seleccionada referenciaron como un tópico de importancia crítica lo relativo cómo obtener financiamiento con un 100\%, mismo porcentaje reflejado en el tema de trato con clientes, ligeramente por debajo se encontró el tema de trato con proveedores con $96.15 \%$, y el trato con equipos multidisciplinarios con un $57.69 \%$.

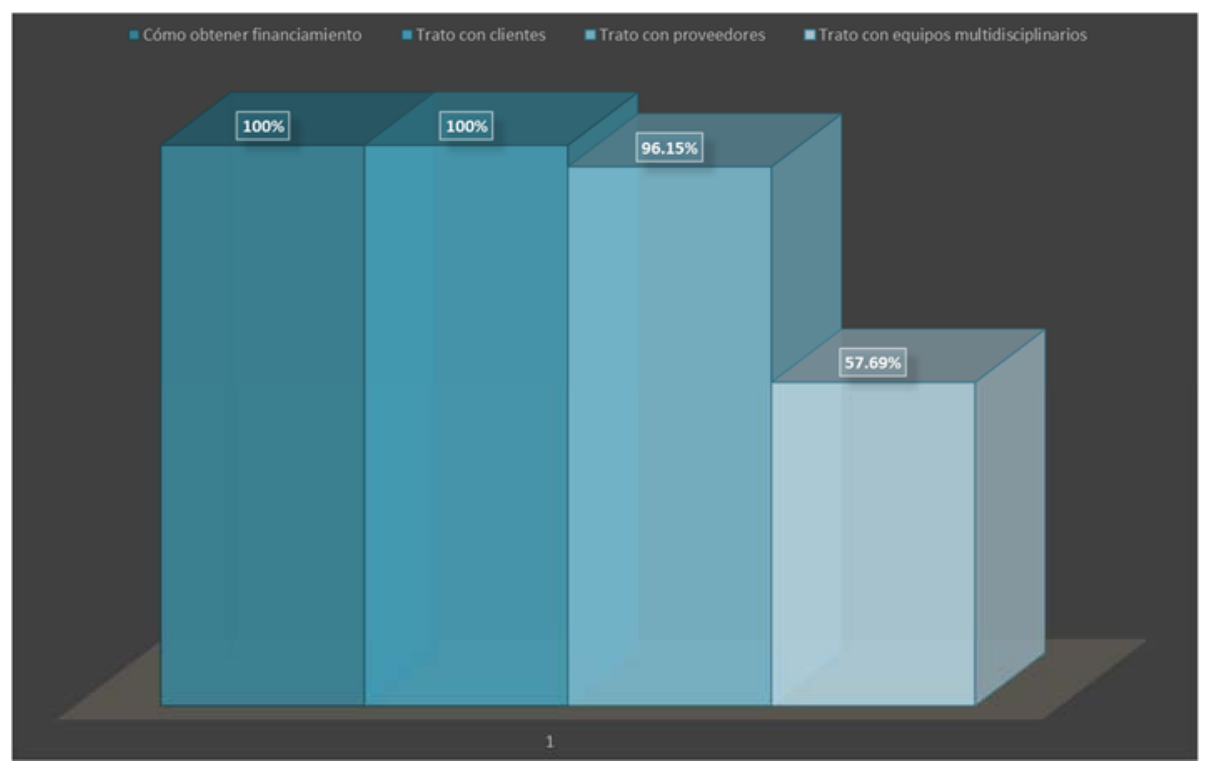

\section{Figura 3. Negociación.}

Fuente: Elaboración propia.

En lo relativo a los aspectos relacionados con la mercadotecnia, estos temas definitivamente ocupan un lugar importante en el perfil óptimo de quienes desean crear una EBT, ya que un tema de fijación de precios o cómo ofrecer soluciones que resultan novedosas en el mercado son temas fundamentales para concretar con éxito la organización.

En la figura 4, los integrantes de la muestra seleccionada referenciaron como un tópico de importancia crítica lo relativo el cómo fijar precios con un $92.31 \%$, seguido con un empate en los temas de cómo ofertar el producto o servicio e identificación de nuevas aplicaciones para su tecnología con un $84.62 \%$ y gestión de marca con un $57.69 \%$. 
CREADORES DE EMPRESAS DE BASE TECNOLÓGICA (SPIN-OFF). IDENTIFICANDO SU PERFIL ÓPTIMO EN MÉXICO.

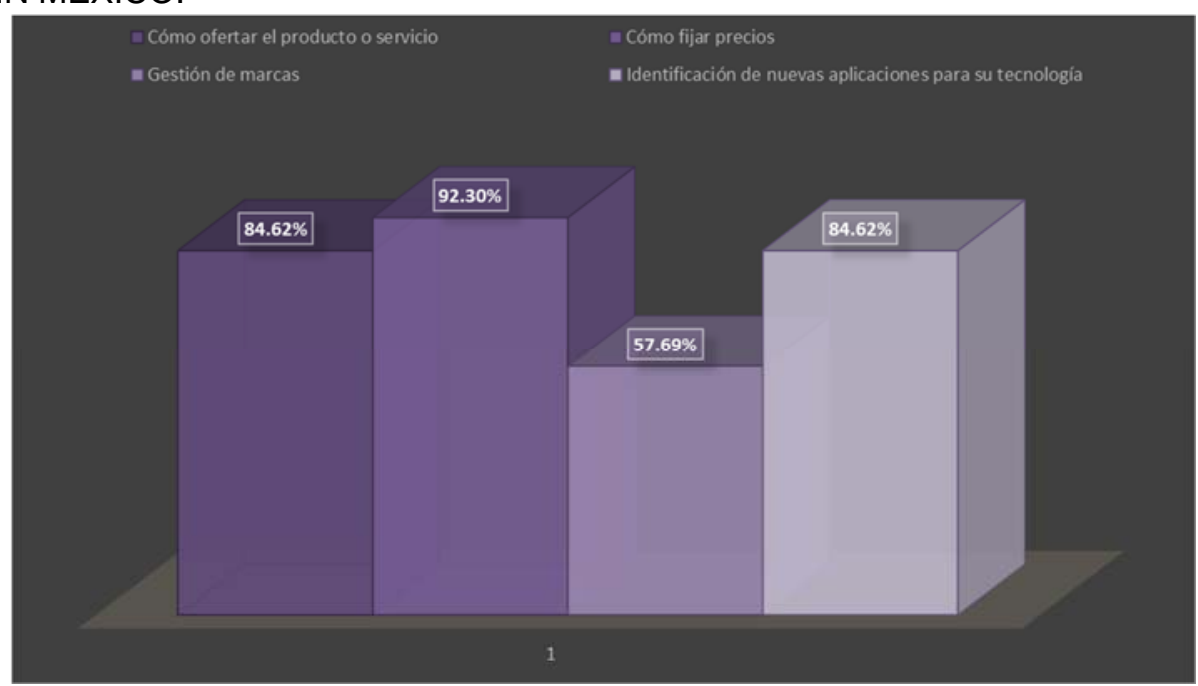

\section{Figura 4. Mercadotecnia.}

Fuente: Elaboración propia.

Ciertamente, el tener una tecnología novedosa que resuelva un problema existente o que pueda desarrollar productos o servicios de gran valor, es una ventaja competitiva indudable; sin embargo, tan importante como lo anterior es la capacidad de cubrir los aspectos legales para consolidar su permanencia en el mercado.

En la figura 5, los integrantes de la muestra seleccionada referenciaron como un tópico de importancia crítica los temas relativo a la gestión de marcas y patentes y a Marco legal de las instituciones de origen (sobre cómo se distribuyen los beneficios obtenidos de las Spin-Off) con un $100 \%$ en ambos casos, seguidos por relaciones con socios con un $88.46 \%$, gestiones laborales con un $65.38 \%$ y gestiones comerciales con un 53.85 .

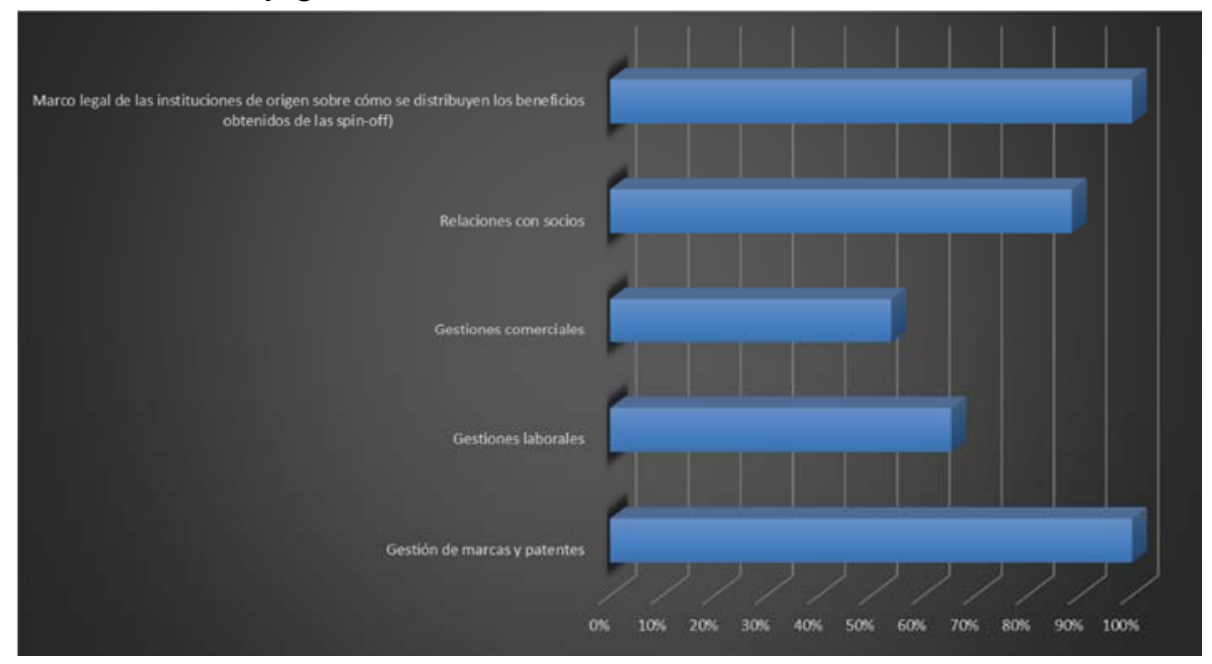

Figura 5. Aspectos legales en general.

Fuente: Elaboración propia. 
CREADORES DE EMPRESAS DE BASE TECNOLÓGICA (SPIN-OFF). IDENTIFICANDO SU PERFIL ÓPTIMO EN MÉXICO.

La apertura y permanencia de las EBT en el mercado debe cubrir los aspectos legales a los que se encuentran sujetos todas las empresas de su giro, por ello, el conocimiento y cumplimiento de las normas y procedimientos es una necesidad insoslayable.

En la figura 6, los integrantes de la muestra seleccionada referenciaron como un tópico de importancia crítica lo relativo tanto a permisos, licencias solicitadas por autoridades como la Secretaría del Trabajo y Previsión Social (STPS), Secretaría de Hacienda y Crédito Público (SHCP), Protección Civil, y los requisitos legales que deben cubrir sus instalaciones con un 100\%, en tanto que los requisitos relativos a higiene, seguridad y ecología con un $84.62 \%$.

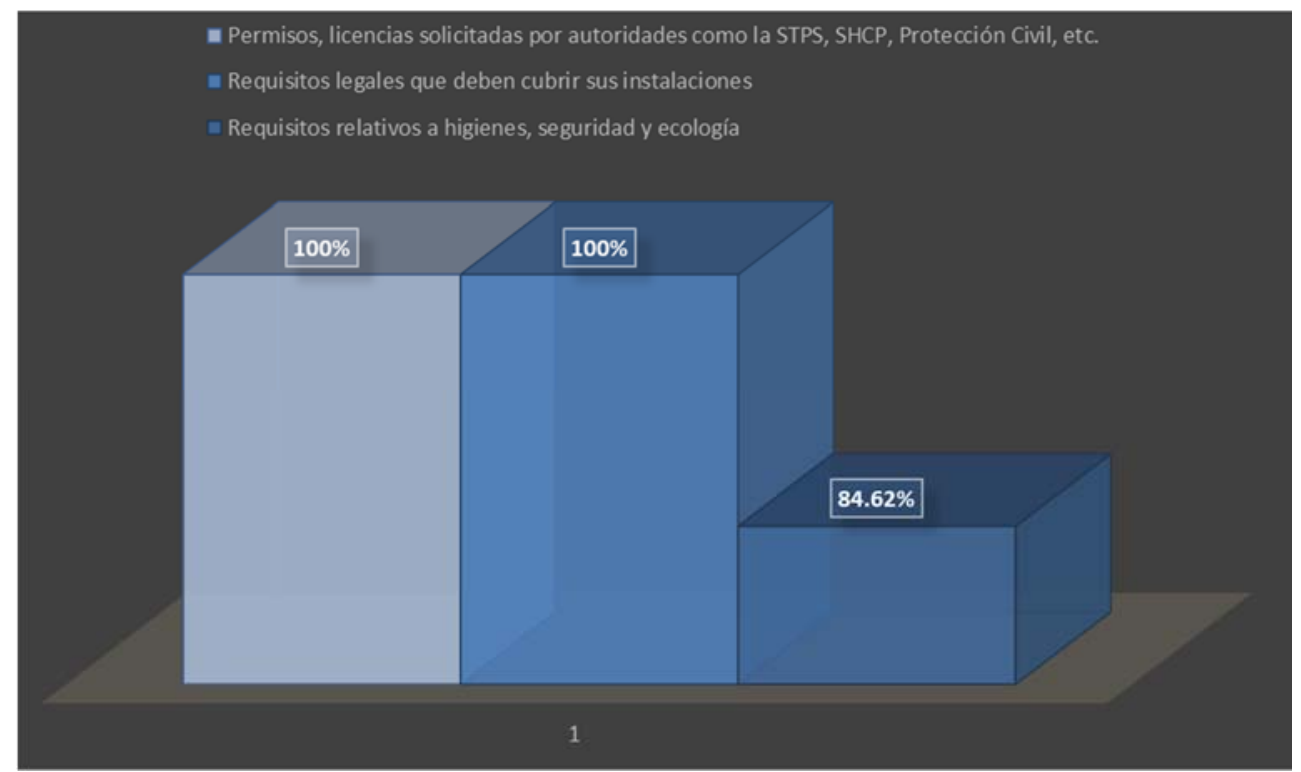

Figura 6. Requisitos legales para poner en marcha un negocio.

Fuente: Elaboración propia.

El cumplimiento de las normas y requisitos legales para los productos y servicios de las EBT son simplemente una necesidad sine qua non (sin la cual no) pueden comercializarse, por ello, resulta indispensable que los creadores de estas empresas conozcan a fondo todos los requisitos que deben cumplir.

En la figura 7, los integrantes de la muestra seleccionada empataron en la importancia de los temas relativos los requisitos legales específicamente lo necesario para cumplir con los requisitos de autoridades como Comisión Federal para la Protección contra Riesgos Sanitarios, Secretaría de Agricultura y Desarrollo Rural) y los requisitos para exportar con un $84.62 \%$, respectivamente. 
CREADORES DE EMPRESAS DE BASE TECNOLÓGICA (SPIN-OFF). IDENTIFICANDO SU PERFIL ÓPTIMO EN MÉXICO.

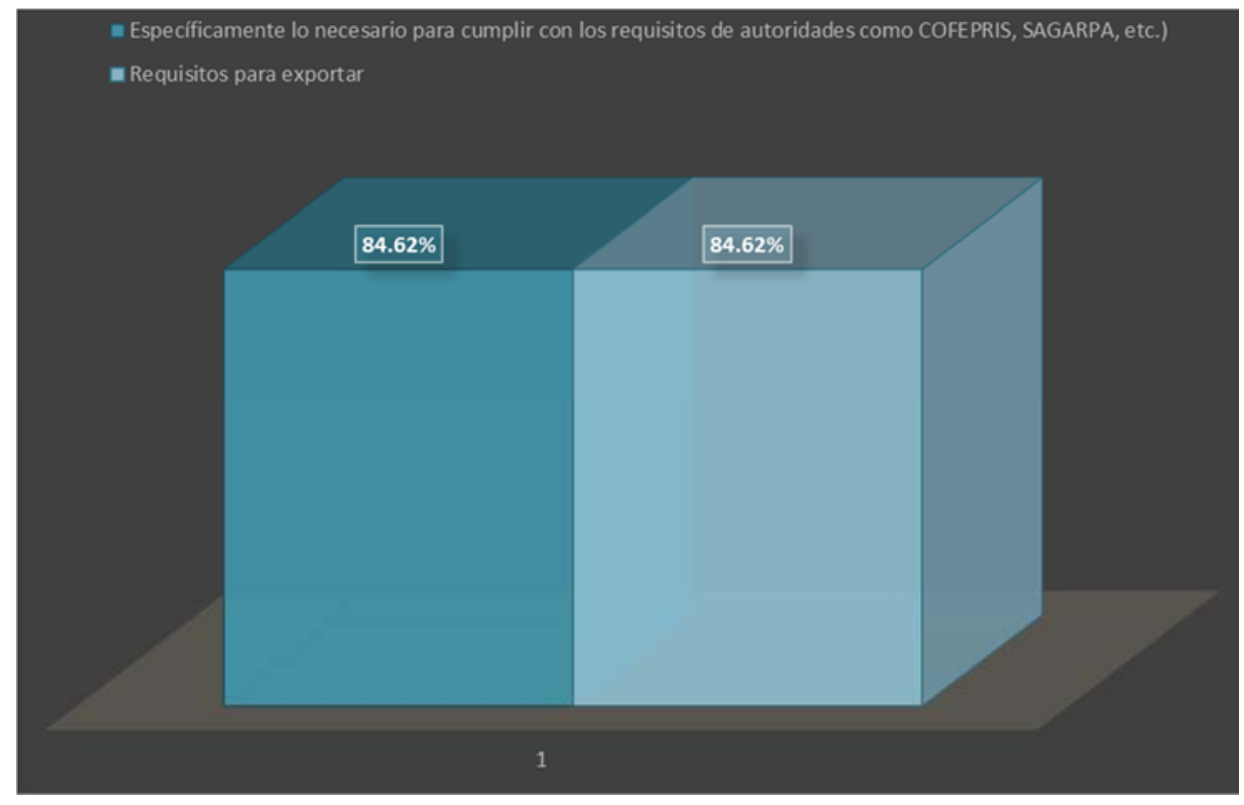

\section{Figura 7. Requisitos legales del producto o servicio.}

Fuente: Elaboración propia.

Es importante mencionar, que en la actualidad existen diferentes universidades que están implementando programas donde sus áreas de administración y legales trabajan en conjunto con las áreas de ciencia, para lograr esquemas donde cada uno hace la parte en la que es experto para consolidar la ETB.

\section{CONCLUSIONES}

Se logró el objetivo, ya que fue posible identificar un perfil óptimo para quienes crean EBT en México; además, fue posible identificar nueve elementos que coadyuvarán para que quienes emprenden o dirigen una EBT, logren una gestión exitosa siendo estos temas relativos a recursos humanos, comunicación efectiva, administración del tiempo, negociación, desarrollo de planes de negocio, mercadotecnia, aspectos legales en general, requisitos legales para poner en marcha un negocio, requisitos legales del producto o servicio.

Seis de los nueve elementos identificados, presentaron sub temas que fueron identificados como críticos para el éxito de las EBT; asimismo, se identificaron los temas que más recurrentemente causan complicaciones a los creadores de EBT tienen que ver con el marco legal, trámites, negociación y recursos humanos, entre otros. 
CREADORES DE EMPRESAS DE BASE TECNOLÓGICA (SPIN-OFF). IDENTIFICANDO SU PERFIL ÓPTIMO EN MÉXICO.

De la misma manera, se identificó la importancia de capacitar a los creadores de EBT en los nueve factores propuestos en el perfil óptimo y la importancia de hacer programas que permitan que las escuelas, institutos, facultades y organismos generadores de tecnología capaces de dar lugar a una EBT tengan contacto y apoyo de las facultades de Administración, Contaduría y Derecho, entre otras, para allanar el camino relativo a la creación y consolidación de la EBT.

Por último, se identificó la importancia de tener un marco sólido de cómo se repartirán las utilidades entre quienes crean las EBT, las instituciones de origen y otros inversores y participantes.

\section{REFERENCIAS BIBLIOGRÁFICAS}

Centro Europeo de Empresas e Innovación. (2012). Manual para la creación de spin-off. Recuperado de https://www.camaracr.org/uploads/tx_icticontent/Manual_Spinoff_01.pdf

Dutta, S., Lanvin, B. y Wunsch-Vincent, S. (Edits.). (2019). Global innovation index 2019 (12th ed.). Recuperado de https://www.wipo.int/edocs/pubdocs/en/wipo_pub_gii_2019.pdf

García, F., Ramírez, G., González, O. y Patricia, L. (2017). Coherencia estratégica de las spin-off universitarias y su impacto en el contexto mexicano. Retos de la Dirección, 11(2), 38-55. Recuperado de http://scielo.sld.cu/scielo.php?script=sci_arttext\&pid=S230691552017000200004

Instituto Mexicano de la Propiedad Industrial. (19 de octubre de 2018). Instituto mexicano de la propiedad industrial en cifras (IMPI en cifras). Recuperado de https://www.gob.mx/impi/documentos/instituto-mexicano-de-la-propiedad-industrial-encifras-impi-en-cifras

Monge, R., Leiva, J. y Alegre, J. (2011). Creación de empresas por parte de ex empleados de empresas multinacionales de alta tecnología en Costa Rica. Tec Empresarial, 5(3), 8-20.

Organización para la Cooperación y el Desarrollo Económicos. (2018). Frascati manual 2015. Guía para la recopilación y presentación de información sobre la investigación y el desarrollo experimental. (F. Fundación Española para la Ciencia y la Tecnología, Ed.) DOI 10.1787/9789264239012-en 


\section{Original}

CREADORES DE EMPRESAS DE BASE TECNOLÓGICA (SPIN-OFF). IDENTIFICANDO SU PERFIL ÓPTIMO EN MÉXICO.

Organización Para La Cooperación Y El Desarrollo Económicos. (2012). Evaluación de la OCDE del sector de las nuevas empresas basadas en el conocimiento. Recuperado de http://www.oecd.org/centrodemexico/Evaluaci\%C3\%B3n_de_la_OCDE_del_sector_de _las_nuevas_empresas_\%20IMPRENTA-1.pdf

Sanchéz, G. (2016). El papel de la propiedad intelectual en la creación de spin-off. Alianzas y Tendencias, 1(3), 6-8. Recuperado de http://www.ditco.buap.mx/recursos/documentos/revista/revista_tres.pdf

World Intellectual Property Organization. (2018). World Intellectual Property Indicators 2018. Recuperado de https://www.wipo.int/edocs/pubdocs/en/wipo_pub_941_2018.pdf 